\title{
INDEX TO THE BIBLIOGRAPHY SUBJECTS AND COUNTRIES
}

\section{GENERAL ISSUES}

Social Theory and Social

Science, 140ff 292ff 469f

History, 142ff., 296ff., 470ff. United States of America,

Contemporary Issues, 149, 154ff., 302ff., $475 \mathrm{ff}$. 298

\author{
CONTINENTS AND COUNTRIES \\ Africa, 149f., 299 \\ Egypt, 473 \\ Ethiopia, 150 \\ Kenya, 150 \\ Lesotho, 473 \\ South Africa, 150f., $473 f$. \\ Tanzania, 300 \\ Zambia, 151
}

America, 151f., 300f., 474

Bolivia, 152

Brazil, 152, 301

Canada, 152, $474 \mathrm{f}$.

Chile, $152 \mathrm{f}$.

Costa Rica, $301 f$.
Cuba, 153, 475

Guadeloupe, 475

Jamaica, 302

Asia, 157f., 478f.

China, 158, 305f., 479

India, 158ff., 306f., $479 \mathrm{f}$.

Indonesia, $480 \mathrm{f}$.

Iran, 481

Israel, 160, 307, 481

Korea, 160, 482

Malaysia, 307

The Philippines, 482

Syria, 482

\section{Australia and Oceania}

Australia, 160f.

New Zealand, 307

Europe, 161, 308f., $482 \mathrm{ff}$.

Austria, 162f., 309, 485f.

Belgium, 163, 309
Czechoslovakia, 163f., 310, 486

Denmark, 310

Eire - Ireland, 164, 310

France, 164ff., 310ff., $486 \mathrm{ff}$.

Germany, 167ff., 314ff., $491 f$.

Great Britain, 171ff., 320ff., 496ff.

Greece, 176, $499 \mathrm{f}$.

Hungary, 176, $324 f$.

Iceland, 177, 500

Italy, 177ff., 325, 500f.

The Netherlands, 179f., 325, $501 f$.

Poland, 325f.

Portugal, 180, 326

Rumania, 326

Spain, 180f., 326f., $502 f$.

Switzerland, 182

Union of Soviet Socialist Republics - Russia, 182f., 327f., $503 f f$.

Yugoslavia, 328 